FIAN-TD-2013-10

\title{
On higher spin extension of the Jackiw-Teitelboim gravity model
}

\author{
K.B. Alkalaev in \\ I.E. Tamm Department of Theoretical Physics, \\ P.N. Lebedev Physical Institute, \\ Leninsky ave. 53, 119991 Moscow, Russia
}

\begin{abstract}
We formulate $A d S_{2}$ higher spin gravity as BF theory with fields taking values in $\operatorname{sl}(N, \mathbb{R})$ algebra treated as higher spin algebra. The theory is topological and naturally extends the Jackiw-Teitelboim gravity model so as to include higher spin fields. The BF equations linearized about $A d S_{2}$ background are interpreted as describing higher spin partially-massless fields of maximal depth along with dilaton fields. It is shown that there are dual metric-like formulations following from the original linearized BF higher spin theory. The duality establishes a dynamical equivalence of the metric-like field equations that can be given either as massive scalar field equations or as conservation conditions for higher spin currents.
\end{abstract}

\section{Introduction}

Two-dimensional gauge systems including generally covariant theories and those with higher rank fields strike a balance between descriptive simplicity and nontrivial content. Basically, this can be explained by that in two dimensions, unlike in higher dimensions, only scalar or spinor modes can propagate. It follows that some of gauge systems are topological while others may exhibit a quite complicated dynamical structure which encodes matter modes. Both cases are intrinsically related because the matter can interact via topological gauge fields, while matter modes themselves can be represented as particular components of gauge fields. This is most clearly illustrated by the massive Schwinger model of fermions interacting via topological Maxwell field, or by the Liouville field originating as the metric component in the conformal gauge.

\footnotetext{
${ }_{\dagger}^{\dagger}$ mail: alkalaev@lpi.ru
} 
A prominent example of topological gauge systems is the Jackiw-Teitelboim gravity model [1, 2] which replaces the standard gravitation theory. Recall that in two dimensions Einstein tensor vanishes identically so that the simplest alternative is given by diffeomorphism invariant equation of motion of the form 1

$$
R-\Lambda=0
$$

where $R$ is the Ricci scalar curvature built of the metric field $g_{m n}(x)$, while $\Lambda$ is the cosmological constant. I The above theory is obviously non-Lagrangian since one has a single equation for three variables. Adding an auxiliary scalar field $\phi(x)$ allows one to introduce the variational principle so that the resulting local action describes a particular dilaton gravity. The theory is however topological and contains no local degrees of freedom. At the same time, $A d S_{2}$ spacetime and black hole type objects are solutions of the Jackiw-Teitelboim model [3, 团.

The main purpose of this paper is to elaborate a higher spin generalization of pure gravity system governed by equation (11.1). We introduce higher rank gauge fields and their equations of motion in such a way that the resulting gauge system contains the gravitational subsector. As a guiding principle, we employ the frame-like formulation instead of the metric-like one used to postulate equation (1.1). The idea behind the framelike approach originates from the Cartan formulation of the Einstein gravity where the metric is the symmetric component of the vielbein, while the antisymmetric component is a pure gauge by local Lorentz transformations.

Indeed, when extending to higher spins, the Jackiw-Teitelboim model viewed as a gauge theory of the $A d S_{2}$ global symmetry algebra $o(2,1) \sim s l(2, \mathbb{R})$ with a BF local action [5] has conceptual advantages over alternative approaches. One considers BF action with fields taking values in $\operatorname{sl}(N, \mathbb{R})$ algebra with a finite parameter $N$. We show that such a theory can be interpreted as two-dimensional topological $A d S_{2}$ higher spin gravity theory. Note that conventional higher-spin metric-like fields $g_{m_{1} \ldots m_{s}}$ with $s$ Lorentz indices appear as particular tensor components of $s l(N, \mathbb{R})$ gauge connection rewritten in $o(1,1) \subset o(2,1) \subset \operatorname{sl}(N, \mathbb{R})$ manifestly Lorentz covariant basis. Other components are either auxiliary or pure gauges. We find that the set of local fields over $A d S_{2}$ background consists of gauge fields with spins $s=2,3, \ldots, N$ and masses $m_{s}^{2}=s(s-1) \Lambda$ interpreted as topological partially-massless fields of maximal depth. [3

On going from the original $\mathrm{BF}$ formulation linearized over $A d S_{2}$ space to the metric-like formulation one finds out that there are two different but dynamically equivalent forms

\footnotetext{
${ }^{1}$ Manifold $\mathcal{M}^{2}$ is a general two-dimensional space-time with local coordinates $x^{m}$, Lorentz world indices run $m, n=0,1$, Lorentz fiber indices run $a, b=0,1, o(2,1)$ fiber indices run $A, B, C=0,1,2$, $o(2,1)$ invariant metric is $\eta^{A B}=(+--)$. The Levi-Civita tensor $\epsilon_{A B C}$ is normalized as $\epsilon_{012}=+1$. Twodimensional anti-de Sitter spacetime $A d S_{2}$ has a radius $L$ and a signature (+-), so that the cosmological constant is $\Lambda=-1 / L^{2}$. The Levi-Civita tensor $\epsilon_{m n}$ is normalized as $\epsilon_{01}=+1$.

${ }^{2}$ The Riemann tensor in two dimensions has only a scalar curvature component as one can see from the identity $\epsilon^{m n} \epsilon^{k l} R_{m n, k l}=2 R$.

${ }^{3}$ The dilaton sector comprising 0 -form fields of the $\mathrm{BF}$ formulation will be analyzed elsewhere [16].
} 
of the metric-like theory. In particular, it turns out that the gauge sector of the quadratic theory can be dually represented either by massive scalar equations or by conservation conditions for higher spin currents. Both scalar/current equations are invariant with respect to on-shell symmetries/improvements that guarantee the absence of local degrees of freedom.

While the BF theories under consideration are topological, we have in mind to study $A d S_{2}$ models with local degrees of freedom interacting via higher spin topological fields. Such an interacting theory will contain at least cubic field-current interactions of the form $\sum_{s} \int d^{2} x \sqrt{g_{A d S_{2}}} \Omega_{m}{ }^{a_{1} \ldots a_{s}} J^{m}{ }_{a_{1} \ldots a_{s}}$, where $\Omega_{m}$ are some Lorentz spin-s gauge fields, while $J_{m}$ are conserved Lorentz spin-s currents built of matter fields. He suggest that the field-current duality mentioned above will play an important role in the analysis of the higher spin field-current couplings in $A d S_{2}$ space. It follows that one of the aims of the present paper is to classify possible higher spin gauge fields in $A d S_{2}$ space that may couple to conserved higher spin currents.

\section{The BF higher spin extension}

Gravitational fields in two dimensions can be identified with $o(2,1)$ connection $W^{A}(x) T_{A}=$ $d x^{m} W_{m}^{A}(x) T_{A}$, where $T_{A}$ are $o(2,1)$ basis elements with the commutation relations $\left[T_{A}, T_{B}\right]=$ $-\epsilon_{A B C} T^{C}$. 囷 Adding 0 -form fields $\Psi(x)=\Psi^{A}(x) T_{A}$ one arrives at the BF action [5]

$$
S_{J T}[W, \Psi]=\int_{\mathcal{M}^{2}} \Psi_{A} \mathcal{R}^{A},
$$

where the curvature is given by $\frac{1}{2} d x^{m} \wedge d x^{n} \mathcal{R}_{m n}^{A} \equiv \mathcal{R}^{A}=d W^{A}-\epsilon^{A B C} W_{B} \wedge W_{C}$. The equations of motion following from (2.1) are

$$
\mathcal{R}_{m n}^{A}=0, \quad D_{m} \Psi^{A}=0,
$$

where $D_{m}=\partial_{m}+W_{m}$ is the $o(2,1)$ covariant derivative. The 1-form connection denoted as $W_{0}^{A}=\left(\sqrt{-\Lambda} h_{m}^{a}, w_{m}\right)$ corresponds to $A d S_{2}$ spacetime. The zero-curvature constraint $\mathcal{R}_{m n}^{A}\left(W_{0}\right)=0$ expresses Lorentz spin connection $w_{m}$ via the zweibein $h_{m}^{a}$, while the latter yields $A d S_{2}$ spacetime metric $g_{m n}$ through the standard identification $g_{m n}=\eta_{a b} h_{m}^{a} h_{n}^{b}$, where the fiber Minkowski metric is $\eta_{a b}=(+-)$.

The BF frame-like equations of motion are dynamically equivalent to the metric-like equations of original Jackiw-Teitelboim model. In particular, the original equation (1.1)

\footnotetext{
${ }^{4}$ A particular example of such a $d=2$ higher spin model has been proposed by Vasiliev [6]. Remarkably, it is formulated as BF theory with fields taking values in some infinite-dimensional algebra, and up to now this is the only example of fully non-linear higher spin gauge theory with local degrees of freedom formulated at the action level.

${ }^{5}$ Note that the basis elements can be equivalently represented in the dualized form as $T^{A}=\frac{1}{2} \epsilon^{A B C} T_{B C}$. The commutation relations for antisymmetric basis elements $T^{A B}=-T^{B A}$ take the standard form $\left[T^{A B}, T^{C D}\right]=\eta^{A D} T^{B C}+3$ terms.
} 
is identified with one component of the curvature (2.2), while other two components are constraints (see [5] for more details). Despite the general covariance, the theory describes a scalar Liouville mode that follows from equation (1.1) in the conformal gauge. However, the theory is topological because the resulting Liouville equation possesses a residual coordinate invariance that gauges away all functional freedom of the solutions, see, e.g., [7].

The higher spin generalization of the theory (2.1) is straightforward. Two-dimensional BF theory with $\mathcal{A}$-valued fields, where $\mathcal{A}=\operatorname{sl}(N, \mathbb{R})$ can be considered as particular higher spin gauge theory. In order to introduce higher spin gauge fields one decomposes the adjoint of $\operatorname{sl}(N, \mathbb{R})$ algebra into totally symmetric irreps of $A d S_{2}$ spacetime global symmetry algebra $\operatorname{sl}(2, \mathbb{R}) \approx o(2,1)$. For instance, in the case of $\mathcal{A}=\operatorname{sl}(3, \mathbb{R})$ the basis elements $T^{\alpha}$ labelled by $\alpha=1, \ldots, 8$ can be equally rearranged as $T^{A} \oplus T^{(A B)}$ with $A, B=$ $0,1,2$, i.e., as a direct sum of rank-1 and rank- 2 totally symmetric and traceless $\operatorname{sl}(2, \mathbb{R})$ algebra tensors. In particular, respective one-form connections are identified with framelike fields of spins 2 and 3. The analogous construction has been used in the context of $3 d$ Chern-Simons higher spin theory with algebra $\mathcal{A} \oplus \mathcal{A}$, where $\mathcal{A}=\operatorname{sl}(3, \mathbb{R})$ [8, 9].

It follows that Lie algebra $\operatorname{sl}(N, \mathbb{R})$ for $N=2,3, \ldots$ can be interpreted as a finitedimensional higher spin algebra in two dimensions provided that its basis elements are organized as follows

$$
T_{A_{1}} \oplus T_{A_{1} A_{2}} \oplus \cdots \oplus T_{A_{1} \ldots A_{N-1}},
$$

where $T_{A_{1} \ldots A_{k}}$ are rank- $k$ totally symmetric and traceless $s l(2, \mathbb{R})$ algebra tensors,

$$
T_{\left(A_{1} \ldots A_{k}\right)}: \quad \eta^{M N} T_{M N A_{3} \ldots A_{k}}=0
$$

It is worth noting that the decomposition (2.3) comes from the principal embedding of $\operatorname{sl}(2, \mathbb{R}) \subset \operatorname{sl}(N, \mathbb{R})$. f

Introduce differential 0 -form and 1 -form fields taking values in $\mathcal{A}=\operatorname{sl}(N, \mathbb{R})$ with basis elements (2.3)

$$
\Psi(x)=\sum_{s=2}^{N-1} \Psi^{A_{1} \ldots A_{s-1}}(x) T_{A_{1} \ldots A_{s-1}}, \quad W(x)=\sum_{s=2}^{N-1} d x^{m} W_{m}^{A_{1} \ldots A_{s-1}}(x) T_{A_{1} \ldots A_{s-1}} .
$$

Expansion coefficients in the higher spin algebra basis elements (2.3) are to be identified as higher spin fields. They satisfy $o(2,1)$ irreducibility conditions of the form (2.4).

Let us define $A d S_{2}$ higher spin gravity by the following BF action

$$
S[W, \Psi]=g \sum_{s=2}^{N-1} \int_{\mathcal{M}^{2}} \Psi_{A_{1} \ldots A_{s-1}} \mathcal{R}^{A_{1} \ldots A_{s-1}},
$$

\footnotetext{
${ }^{6} \mathrm{~A}$ non-principal embedding gives rise to a different dynamical content, and, generally speaking, to a different theory. In the context of $3 d$ Chern-Simons higher spin theory non-principal embeddings and respective spectra were discussed, e.g., in [10].
} 
with curvature two-forms given by $\mathcal{R}_{m n}^{A_{1} \ldots A_{s-1}}=\partial_{[m} W_{n]}^{A_{1} \ldots A_{s-1}}+\left[W_{m}, W_{n}\right]^{A_{1} \ldots A_{s-1}}$, where $[\cdot, \cdot]$ is the Lie bracket in $\mathcal{A}$ evaluated in basis (2.3), and $g$ is a dimensionless coupling constant. The action (2.6) is invariant under the $\operatorname{sl}(N, \mathbb{R})$ gauge transformations

$$
\delta W_{m}^{A_{1} \ldots A_{s-1}}=\partial_{m} \xi^{A_{1} \ldots A_{s-1}}+\left[\xi, W_{m}\right]^{A_{1} \ldots A_{s-1}}, \quad \delta \Psi^{A_{1} \ldots A_{s-1}}=[\xi, \Psi]^{A_{1} \ldots A_{s-1}}
$$

where $\xi(x)=\sum_{s=2}^{N-1} \xi^{A_{1} \ldots A_{s-1}}(x) T_{A_{1} \ldots A_{s-1}}$ is an $\mathcal{A}=\operatorname{sl}(N, \mathbb{R})$-valued gauge parameter. Note that a-priori there is no metric tensor as the theory is completely formulated via differential forms. It follows that the theory is diffeomorphism invariant. However, an on-shell diffeomorphism transformation is simply a gauge transformation with particular gauge parameter.

Variational equations of motion for $\Psi^{A_{1} \ldots A_{s-1}}$ and $W_{m}^{A_{1} \ldots A_{s-1}}$ are the zero-curvature equation and the covariance constancy equation

$$
\mathcal{R}_{m n}^{A_{1} \ldots A_{s-1}}=0, \quad D_{m} \Psi^{A_{1} \ldots A_{s-1}}=0, \quad s=2, \ldots, N-1
$$

where $D_{m}=\partial_{m}+W_{m}$ is a covariant derivative with 1-form connection $W_{m}$ given by (2.5). The covariance constancy equation involves both 0 -form and 1-form fields, while the zero-curvature equation forms an independent gauge sector of the theory that can be analyzed separately. Let us emphasize that solutions of the zero-curvature equation are given locally by pure gauge 1-form fields. For the case of finitely many gauge fields this observation guarantees that the theory is topological and therefore contains no local degrees of freedom. However, in the next section we explicitly examine the gauge sector in the free field approximation to provide a particular physical interpretation of topological modes.

\section{$3 \quad$ Linearized higher spin dynamics in $A d S_{2}$ space}

The higher spin theory (2.6) contains a gravitational sector described by the JackiwTeitelboim action. The gravitational fields are singled out by setting all higher spin fields to zero except for those taking values in $\operatorname{sl}(2, \mathbb{R})$ subalgebra. This particular truncation is obviously consistent. At the same time, $A d S_{2}$ space is the ground state of the theory since it solves gravitational equations (1.1).

Since the action (2.6) is non-linear containing at most cubic terms $\sim \Psi W^{2}$, one can consider the perturbation theory over a particular solution. To this end introduce 1-form $W_{0}$ describing $A d S_{2}$ spacetime and 0 -form $\Psi_{0}=0$ as the background of the theory, so that all fields are decomposed as $W=W_{0}+\Omega$ and $\Psi=\Psi_{0}+\Phi$, where $\Omega$ and $\Phi$ are fluctuations over the background. The background fields $W_{0}$ and $\Psi_{0}$ obviously solve BF higher spin equations (2.8). Similarly, the 2 -form curvatures are expanded as $\mathcal{R}=\mathcal{R}_{0}+R+\ldots$, where $\mathcal{R}_{0} \equiv \mathcal{R}\left(W_{0}\right)$ is the zero-curvature gravitational constraint (2.2), while $R$ is the linearized curvature and the ellipsis denote second order corrections. 
In the one-form sector, gauge fields $\Omega$ and their linearized curvatures $R$ have the component form

$$
\Omega^{A_{1} \ldots A_{s-1}}=d x^{m} \Omega_{m}^{A_{1} \ldots A_{s-1}}, \quad R^{A_{1} \ldots A_{s-1}}=D_{0} \Omega^{A_{1} \ldots A_{s-1}}
$$

where $D_{0}=d+W_{0}$ is the background covariant derivative. Representing the zerocurvature condition (2.2) as $\mathcal{R}\left(W_{0}\right) \equiv D_{0}^{2}=0$ one obtains that linearized curvatures are invariant under the gauge transformations (2.7) expanded over the background,

$$
\delta \Omega^{A_{1} \ldots A_{s-1}}=D_{0} \xi^{A_{1} \ldots A_{s-1}}
$$

where $\xi^{A_{1} \ldots A_{s-1}}$ are 0 -form gauge parameters. The Bianchi identities $D_{0} R^{A_{1} \ldots A_{s-1}} \equiv 0$ following from (3.1) are trivial since any 3 -form in two dimensions vanishes identically. Fluctuations in the zero-form sector are inert under the gauge transformations $\delta \Psi^{A_{1} \ldots A_{s-1}}=0$, at least to the lowest order in the fields, cf. (2.7).

Let us compare the resulting set of 1 -form higher spin gauge fields (3.1) in $d=2$ dimensions with fields in $d \geq 3$ dimensions. By massless higher spin fields in $A d S_{d}$ we call 1-form connections $\Omega_{m}^{A_{1} \ldots A_{s-1}, B_{1} \ldots B_{s-1}}$ taking values in $o(2, d-1)$ finite-dimensional irreps of the symmetry type described by two-row rectangular Young diagram [11]. In the metric-like formulation these fields reduce to Fronsdal spin- $s$ massless fields. Obviously, for $d=2$ all 1 -form connections vanish identically except for $s=2$ case of the gravity, $\Omega_{m}^{A_{1}, B_{1}}=-\Omega_{m}^{B_{1}, A_{1}}$. It follows that there are no spin $s>2$ Fronsdal fields in $A d S_{2}$.

At the same time, in two dimensions fiber indices of spin-2 connection can be dualized by virtue of $o(2,1)$ Levi-Civita tensor as $\Omega_{m}^{A_{1}}=\epsilon^{A_{1} M N} \Omega_{m M, N}$. The point is that the gravitational connection $\Omega_{m}^{A}$ starts an infinite sequence of 1 -form connections $\Omega_{m}^{A_{1} \ldots A_{s-1}}$, $s=2,3, \ldots$, taking values in $o(2,1)$ finite-dimensional irreps described by one-row Young diagrams. These are fields appearing via the gauging of $\operatorname{sl}(N, \mathbb{R})$ algebra in the basis (2.3). Note that in the $d \geq 3$ case, the connections with totally symmetric $o(d-1,2)$ fiber indices describe partially-massless fields of maximal depth [12, 13, 14]. It follows that in $A d S_{2}$ space all possible higher spin $s>2$ fields are exhausted by "topological partially-massless" fields of maximal depth. I

The BF equations (2.8) linearized around $A d S_{2}$ background space decouple into a direct sum of $N-1$ independent subsectors

$$
\begin{aligned}
& D_{0} \Omega^{A_{1} \ldots A_{s-1}}=0, \\
& D_{0} \Phi^{A_{1} \ldots A_{s-1}}=0,
\end{aligned}
$$

each of spin $s=2,3, \ldots, N$. In what follows, we analyze a set of topological metric-like fields encoded in the gauge sector described by equations (3.3).

\footnotetext{
${ }^{7}$ In higher dimensions, the depth is a number of derivatives in the gauge transformation law for a spin- $s$ dynamical field, $t=1, \ldots, s$. In two dimensions, a maximal value of the depth equals $t=s-1$ due to the Hodge duality mentioned above.
} 
To identify the dynamical content of equations (3.3) one decomposes $o(2,1)$ covariant gauge fields and linearized curvatures (3.1) into Lorentz algebra $o(1,1) \subset o(2,1)$ irreducible components,

$$
\Omega_{m}^{A_{1} \ldots A_{s-1}}=\bigoplus_{k=0}^{s-1} \omega_{m}^{a_{1} \ldots a_{k}}, \quad R_{m n}^{A_{1} \ldots A_{s-1}}=\bigoplus_{k=0}^{s-1} R_{m n}^{a_{1} \ldots a_{k}}
$$

i.e., totally symmetric and traceless with respect to the Minkowski tensor $\eta^{a b}$. Assuming that Lorentz indices $a_{i}$ are symmetrized with a unit weight, we find that the component form of (3.1) is given by

$$
\begin{aligned}
& R_{m n}^{a_{1} \ldots a_{k}}=\nabla_{[m} \omega_{n]}^{a_{1} \ldots a_{k}}-\Lambda \frac{(s-k-1)(s+k)}{2(k+1)} h_{[m, c} \wedge \omega_{n]}^{c a_{1} \ldots a_{k}}+ \\
& \quad+\left[h_{[m}^{a_{1}} \wedge \omega_{n]}^{a_{2} \ldots a_{k}}-\frac{1}{k-1} \eta^{a_{1} a_{2}} h_{[m, c} \wedge \omega_{n]}^{c a_{3} \ldots a_{k}}\right],
\end{aligned}
$$

where covariant derivative $\nabla_{m}=\partial_{m}+w_{m}$ is defined with respect to the background Lorentz spin connection $w_{m}$, while $h_{m}^{a}$ is the background zweibein. Quite analogously, one finds the component form of the gauge transformations (3.2), where gauge parameters $\xi^{a_{1} \ldots a_{k}}$ are $o(1,1)$ irreducible components of the $o(2,1)$ gauge parameter $\xi^{A_{1} \ldots A_{s-1}}(3.2)$.

The Lorentz component form of BF equations (3.3) is

$$
R_{m n}^{a_{1} \ldots a_{k}}=0, \quad k=0,1, \ldots, s-1 .
$$

In the spin $s=1$ case the only field $\omega_{m}$ is identified with potential $A_{m}$. The resulting BF equation $R_{m n} \equiv F_{m n}=0$ is different form the Maxwell equation $\nabla_{m} F^{m n}=0$. It is worth noting here that a similar difference is between Maxwell and Chern-Simons equations in three dimensions.

The analysis of equations (3.7) for $\operatorname{spin} s>1$ is more intricate because there are more different rank fields and their gauge transformations including both differential and algebraic symmetry parameters. All fields entering equations of motion (3.7) can be divided into three subsets consisting of dynamical fields, Stueckelberg fields, and auxiliary fields. F It turns out that such a triple decomposition of the original field space can be done in two different ways. In the following sections we explicitly demonstrate how it works in the spin-2 case, and then briefly generalize to arbitrary spins.

\subsection{Spin-2 example}

In the spin $s=2$ case fields $\omega_{m}^{a}$ and $\omega_{m}$ are identified with the zweibein and Lorentz spin connection (more precisely, with their fluctuations over $A d S_{2}$ values $h_{m}^{a}$ and $w_{m}$ ). Field

\footnotetext{
${ }^{8}$ As usual, Stueckelberg fields are assumed to be set to zero by virtue of their algebraic gauge transformations, while auxiliary fields are expressed via dynamical fields. Despite the absence of local degrees of freedom we keep using the term "dynamical fields" to designate independent fields of the theory.
} 
equations read off from (3.7) and (3.6) at $s=2$

$$
R_{m n} \equiv \nabla_{m} \omega_{n}-\nabla_{n} \omega_{m}-\Lambda h_{m, b} \wedge \omega_{n}^{b}+\Lambda h_{n, b} \wedge \omega_{m}^{b}=0
$$

and

$$
R_{m n}^{a} \equiv \nabla_{m} \omega_{n}^{a}-\nabla_{n} \omega_{m}^{a}+h_{m}^{a} \wedge \omega_{n}-h_{n}^{a} \wedge \omega_{m}=0,
$$

are invariant under the gauge transformations

$$
\delta \omega_{m}=\nabla_{m} \xi-\Lambda h_{m, c} \xi^{c}, \quad \delta \omega_{m}^{a}=\nabla_{m} \xi^{a}+h_{m}^{a} \xi
$$

Using dualized field redefinitions $\omega_{m}^{a} \equiv \epsilon^{a b} e_{m, b}$ and $\omega_{m} \equiv \epsilon_{a b} \omega_{m}^{[a b]}$, the equations (3.8) and (3.9) can be cast into the standard form for zweibein $e_{m}^{a}$ and Lorentz spin connection $\omega_{m}^{a b}$. In particular, the above field redefinition succeeded by setting $\Lambda=0$ reproduces the known expressions for curvatures of the $(1+1)$ Poincare algebra.

The standard choice of dynamical fields is achieved by using the gauge parameter $\xi$ as the Stueckelberg one to gauge away the trace component of $\omega_{m}^{a}$. of To this end, using the $A d S_{2}$ background frame $h_{m}^{a}$ one converts world indices into fiber ones and then decomposes $\omega_{m}^{a}$ into three $o(1,1)$ irreducible components as follows

$$
\omega^{a \mid b}=\omega^{(a b)}+\eta^{a b} \hat{\omega}+\frac{1}{2} \epsilon^{a b} \tilde{\omega}
$$

where $\eta_{m n} \omega^{(m n)}=0$. Residual components of $\omega_{m}^{a}$ can be combined into a single traceful symmetric tensor $\varphi_{a b}=\epsilon_{a}{ }^{c} \omega_{c b}+\frac{1}{2} \eta_{a b} \tilde{\omega}$, so that its traceless and trace parts are given by $\omega^{(a b)}$ and $\tilde{\omega}$. Field $\varphi^{a b}$ is then identified as dynamical field with the gauge symmetry transformation $\delta \varphi_{a b}=\nabla_{a}\left(\epsilon_{b}{ }^{c} \xi_{c}\right)+\nabla_{b}\left(\epsilon_{a}{ }^{c} \xi_{c}\right)$ (cf. our comments below (3.10)). Simultaneously, one considers equation (3.9) as the constraint which allows one to express auxiliary field $\omega_{m}$ via dynamical field $\varphi^{a b}$.

Equation $\epsilon^{m n} R_{m n}=0$ (3.8) is dynamical. In terms of $\varphi_{a b}$ it reads $\nabla^{a} \nabla^{b} \varphi_{a b}-\frac{3}{2} \square_{A d S_{2}} \varphi+$ $2 \Lambda \varphi=0$, where $\square_{A d S_{2}}$ is the wave operator for the $A d S_{2}$ metric, and $\varphi=\eta^{a b} \varphi_{a b}$. Obviously, this is the Jackiw-Teitelboim equation (1.1) linearized around $A d S_{2}$ background. Imposing the conformal gauge which in our case says that the traceless part of $\varphi^{a b}$ is set to zero, one is left with $\varphi$ subjected to the residual equation

$$
\left(\square_{A d S_{2}}-2 \Lambda\right) \varphi=0
$$

The above equation is invariant under the residual gauge transformation $\delta \varphi=\epsilon_{a b} \nabla^{a} \xi^{b}$ with a parameter satisfying the Killing equation $\nabla^{a} \xi^{b}+\nabla^{b} \xi^{a}-\eta^{a b} \nabla_{c} \xi^{c}=0$. Therefore, one is left with the scalar field which is massive gauge field. To

It is crucial that for $\Lambda \neq 0$ the above choice of dynamical fields is not unique. Another option is to use the gauge parameter $\xi^{a}$ as a Stueckelberg one to completely gauge away

\footnotetext{
${ }^{9}$ Scalar parameter $\xi$ can be dualized into an antisymmetric parameter $\xi^{[a b]}$. Obviously, this choice corresponds to the standard gauge fixing of local Lorentz symmetry.

${ }^{10}$ Recall here the well-known example of a scalar gauge field given by the trace of the metric field.
} 
the field $\omega^{m}(3.10)$. Then, from (3.8) and (3.11) one derives the algebraic constraint $\Lambda \tilde{\omega}=0$, which says that the antisymmetric component of $\omega_{m}^{a}$ (3.11) is set to zero. It follows that a dynamical field is again a traceful symmetric tensor $\phi^{a b}=\omega^{(a b)}+\eta^{a b} \hat{\omega}$, but now its traceless and trace components are identified with $\omega^{(a b)}$ and $\hat{\omega}$. The gauge transformations now read $\delta \phi^{a b}=\Lambda^{-1} \nabla^{a} \nabla^{b} \xi+\eta^{a b} \xi$. Note that it reproduces the gauge transformation of a spin-2 partially-massless field.

Equation $\epsilon^{m n} R_{m n}^{a}=0$ (3.9) is dynamical. Imposing the gauge $\eta_{a b} \phi^{a b}=0$ one is left with a traceless component subjected to the residual equation

$$
\nabla^{a} J_{a b}=0
$$

where $J^{a b} \equiv \epsilon^{a c} \phi^{b}{ }_{c}$ is symmetric and traceless tensor field. A residual gauge invariance of (3.13) is given by $\delta J_{a b}=\Lambda^{-1} \epsilon_{a c} \nabla^{c} \nabla_{b} \xi+\Lambda^{-1} \epsilon_{b c} \nabla^{c} \nabla_{b} \xi$ with the scalar parameter satisfying differential constraint $\left(\square_{A d S_{2}}-2 \Lambda\right) \xi=0$. It follows that equation (3.13) can be seen as the conservation condition for a rank-2 current $J_{a b}$ on the $A d S_{2}$ space.

\subsection{Standard realization}

By the standard realization we mean using a set of dynamical fields that generalize the metric-like variable of the spin-2 case. A careful analysis shows that all higher rank equations in (3.7) are constraints except for the $k=0$ equation $\epsilon^{m n} R_{m n}=0$ treated as the dynamical equation. The constraints allow one to express all fields via derivatives of dynamical fields which are scalar and rank-s traceless tensors, $\varphi$ and $\varphi^{a_{1} \ldots a_{s}}$. As in the spin-2 case, the scalar component $\varphi$ is identified with antisymmetric component of $\omega_{m}^{a}$ (3.11). The rank-s traceless component $\varphi^{a_{1} \ldots a_{s}}$ is identified with a totally symmetrized highest rank $k=s-1$ connection $\varphi^{m a_{1} \ldots a_{s-1}}=\omega^{\left(m \mid a_{1} \ldots a_{s-1}\right)}$. Note that the symmetrized combination is automatically traceless because all other components in $\omega_{m}^{a_{1} \ldots a_{s-1}}$ are gauged away using the Stueckelberg symmetry transformations.

Quite analogously, one shows that the only independent gauge parameter is $\xi^{a_{1} \ldots a_{s-1}}$, while all others are treated as Stueckelberg parameters or auxiliary parameters expressed via derivatives of $\xi^{a_{1} \ldots a_{s-1}}$. The leftover gauge symmetry with parameter $\xi^{a_{1} \ldots a_{s-1}}$ allows one to impose the higher spin gauge,

$$
\varphi^{a_{1} \ldots a_{s}}=0,
$$

which is legitimate since any $o(1,1)$ rank- $k$ irreducible tensor for $k>0$ has just two independent components. Then, the only dynamical field is given by the scalar $\varphi$. Imposing the higher spin gauge (3.14) and solving the constraints in (3.7) one finds that the leftover equation reduces to the massive scalar equation with a particular value of mass-like term

$$
\left(\square_{A d S_{2}}-m_{s}^{2}\right) \varphi=0, \quad \text { where } \quad m_{s}^{2}=s(s-1) \Lambda, \quad s \geq 2 .
$$

This is invariant under the residual gauge transformations,

$$
\delta \varphi=\epsilon_{m n_{1}} \nabla^{m} \nabla_{n_{2}} \cdots \nabla_{n_{s-1}} \xi^{n_{1} \cdots n_{s-1}}
$$


provided that the gauge parameter satisfies the generalized Killing equation $\nabla^{\left(a_{1}\right.} \xi^{\left.a_{2} \ldots a_{s}\right)}-$ $\frac{1}{s-1} \eta^{\left(a_{1} a_{2}\right.} \nabla_{c} \xi^{\left.a_{3} \ldots a_{s-1}\right) c}=0$, which is simply the stability transformation for the higher spin gauge (3.14). The massive scalar field (3.15) endowed with higher order derivative transformations for the scalar parameter (3.16) can be treated as topological partiallymassless field of spin $s$ and the maximal depth.

\subsection{Dual realization}

By the dual realization we mean using a set of dynamical fields that generalize the currentlike variable of the spin-2 case. Quite analogously to the standard realization one shows that all higher rank equations in (3.7) can be treated as constraints except for the $k=s-1$ equation $\epsilon^{m n} R_{m n}^{a_{1} \ldots a_{s-1}}=0$ which is dynamical. The constraints allow one to express all fields via derivatives of dynamical fields also given by scalar and rank- $s$ symmetric traceless tensor, $\phi$ and $\phi^{a_{1} \ldots a_{s}}$. But now the scalar $\varphi$ is identified with a trace component of $\omega_{m}^{a}$ (3.11), while the rank- $s$ traceless field $\phi^{a_{1} \ldots a_{s}}$ is identified with the symmetric traceless component of $k=s-1$ connection $\omega_{m \mid a_{1} \ldots a_{s-1}}$, namely $\phi_{m a_{1} \ldots a_{s-1}}=\omega_{\left(m \mid a_{1} \ldots a_{s-1}\right)}-$ traces. An independent gauge parameter is the scalar $\xi$, while the gauge transformations read

$$
\delta \phi=\square_{A d S_{2}} \xi-s(s-1) \Lambda \xi, \quad \delta \phi_{a_{1} \ldots a_{s}}=\nabla_{a_{1}} \cdots \nabla_{a_{s}} \xi+\ldots
$$

where the ellipsis refers to proper symmetrizations and trace terms. Using gauge symmetry (3.17) one can impose the scalar gauge $\phi=0$ along with the residual gauge parameter equation $\square_{A d S_{2}} \xi-s(s-1) \Lambda \xi=0$.

In the scalar gauge, the only dynamical field is the rank-s component $\phi^{a_{1} \ldots a_{s}}$. Then, solving the constraints in (3.7) one finds that the independent dynamical equation is given by

$$
\nabla^{n} J_{n a_{1} \ldots a_{s-1}}=0 \quad \text { for } \quad J_{n a_{1} \ldots a_{s-1}}=\epsilon_{n}{ }^{m} \phi_{m a_{1} \ldots a_{s-1}},
$$

where dualized tensor field $J_{n a_{1} \ldots a_{s-1}}$ is totally symmetric and traceless. One identifies $J_{a_{1} \ldots a_{s}}$ with spin-s conserved current on the $A d S_{2}$ space, while higher order derivative transformations in (3.17) are "improvements". On the other hand, the "improvement" transformation can treated as the gauge transformation of a spin- $s$ partially massless field of maximal depth.

\section{Conclusions and outlooks}

In this paper we have introduced $A d S_{2}$ higher spin gravity defined via topological BF action with fields taking values in $\operatorname{sl}(N, \mathbb{R})$ algebra. We identified the spectrum of higher spin gauge field fluctuations over $A d S_{2}$ background, and for $\Lambda \neq 0$ found out their dual description in terms of higher rank conserved currents.

Note that the proposed duality between two metric-like formulations guarantees that the two realizations describe the same dynamical system. Indeed, in order to single out in- 
dependent fields and parameters we used Stueckelberg algebraic symmetry and eliminated auxiliary fields of the frame-like formulation. Whence, two metric-like formulations (3.15) and (3.18) coming from the original (linearized) BF system are dynamically equivalent. This type of duality is similar to that one in the WZNW theory with the equations of motions for a group variable $g(x)$ given by a second order field equation $\partial^{m}\left(g^{-1} \partial_{m} g\right)=0$. At the same time, introducing the current $J_{m}=g^{-1} \partial_{m} g$ one equivalently arrives at the first order conservation condition $\partial^{m} J_{m}=0$ (e.g., see [15]).

It is instructive to compare linearizations of $A d S_{2}$ higher spin theory for $\mathcal{A}=\operatorname{sl}(N, \mathbb{R})$ algebra and $3 d$ Chern-Simons higher spin theory for $\mathcal{A} \oplus \mathcal{A}$ algebra. In the $3 d$ case, choosing $A d S_{3}$ spacetime as the background for higher spin fields yields Fronsdal equations of motion for massless gauge fields with spins $s=2, \ldots, N$ that describe, however, no local degrees of freedom [8, 9]. In the $2 d$ case, the same analysis does not yield Fronsdal equations, although the system is also topological. Instead, in the higher spin gauge which generalizes the conformal gauge in the gravity, the dynamical equations in the standard realization are scalar field equations with higher order derivative gauge symmetries guaranteeing the absence of propagating modes. These gauge systems can be interpreted as two-dimensional topological partially-massless fields.

It is remarkable that BF higher spin theory considered in the present paper has been in fact formulated within the unfolded approach to higher spin dynamics. In [16] we consider the theory from the unfolded formulation perspective and use the cohomological technique to analyze BF equations of motion. In particular, independent parameters, fields and equations of the standard and the dual realizations elaborated in sections $\mathbf{3 . 2}$ and 3.3 have a streamlined interpretation as cohomology elements of the so-called $\sigma_{-}$and $\sigma_{+}$nilpotent operators acting in the field space. Also, using the cohomological machinery we analyze equations for 0 -forms which were not considered in the present paper.

The proposed BF higher spin theory can be extended so as to include an infinite tower of higher spin fields provided a gauge algebra $\mathcal{A}$ is chosen to be one of infinite-dimensional higher spin algebras [17, 16, 18]. The resulting theory is also topological. Recall that BF higher spin theory can however have local degrees of freedom provided that fields take values in particular infinite-dimensional associative algebra [6].

Acknowledgements. I am grateful to M. Grigoriev, E. Skvortsov and M.A. Vasiliev for useful comments and discussions. I would like to thank the Galileo Galilei Institute for Theoretical Physics, Florence, Italy, for hospitality during my staying at GGI Workshop on Higher Spins, Strings and Duality, and to the Dynasty foundation for the financial support of the visit. The work is supported by RFBR grant 12-02-31838.

Note added. After the present paper has been submitted to arXiv, we were informed by Soo-Jong Rey that $2 d$ higher spin theories were partly discussed in his talk [18], mainly from the holographic perspective. 


\section{References}

[1] C. Teitelboim, "Gravitation and Hamiltonian Structure in Two Space-Time Dimensions," Phys.Lett. B126 (1983) 41.

[2] R. Jackiw, "Lower Dimensional Gravity", Nucl. Phys. B252 (1985) 343.

[3] M. Cadoni and S. Mignemi, "Nonsingular four-dimensional black holes and the Jackiw-Teitelboim theory," Phys.Rev. D51 (1995) 4319-4329, hep-th/9410041.

[4] M. Spradlin and A. Strominger, "Vacuum states for AdS(2) black holes," JHEP 9911 (1999) 021 hep-th/9904143.

[5] T. Fukuyama and K. Kamimura, "Gauge Theory of Two-dimensional Gravity," Phys.Lett. B160 (1985) 259.

K. Isler and C. Trugenberger, "A Gauge Theory of Two-dimensional Quantum Gravity," Phys.Rev.Lett. 63 (1989) 834.

A. H. Chamseddine and D. Wyler, "Topological gravity in (1+1)-dimensions," Nucl. Phys. B340 (1990) 595-616.

[6] M. A. Vasiliev, "Higher-Spin Gauge Interactions for Matter Fields in Two Dimensions," Phys. Lett. B363 (1995) 51-57, hep-th/9511063.

[7] R. Jackiw, "Gauge theories for gravity on a line," Theor.Math.Phys. 92 (1992) 979-987, hep-th/9206093.

[8] M. Henneaux and S.-J. Rey, "Nonlinear $W_{\text {infinity }}$ as Asymptotic Symmetry of Three-Dimensional Higher Spin Anti-de Sitter Gravity," JHEP 1012 (2010) 007, 1008.4579 .

[9] A. Campoleoni, S. Fredenhagen, S. Pfenninger, and S. Theisen, "Asymptotic symmetries of three-dimensional gravity coupled to higher-spin fields," JHEP 1011 (2010) 007, 1008.4744.

[10] A. Castro, E. Hijano, and A. Lepage-Jutier, "Unitarity Bounds in AdS(3) Higher Spin Gravity," JHEP 1206 (2012) 001, 1202.4467.

[11] M. A. Vasiliev, "Cubic interactions of bosonic higher spin gauge fields in AdS(5)," Nucl. Phys. B616 (2001) 106-162, hep-th/0106200.

[12] S. Deser and A. Waldron, "Partial masslessness of higher spins in (A)dS," Nucl.Phys. B607 (2001) 577-604, hep-th/0103198.

[13] Y.M. Zinoviev, "On massive high spin particles in AdS," hep-th/0108192.

[14] E. D. Skvortsov and M. A. Vasiliev, "Geometric formulation for partially massless fields," Nucl. Phys. B756 (2006) 117-147, hep-th/0601095.

[15] C. R. Nappi, "Some Properties of an Analog of the Nonlinear $\sigma$ Model," Phys.Rev. D21 (1980) 418; E. Fradkin and A. A. Tseytlin, "Quantum Equivalence of Dual Field Theories," Annals Phys. 162 (1985) 31.

[16] K. Alkalaev, "Global and local properties of $A d S_{2}$ higher spin gravity," preprint FIAN-TD-2013-16, to be published. 
[17] E. Fradkin and V. Y. Linetsky, "Higher spin symmetry in one-dimension and two-dimensions, I," Mod.Phys.Lett. A4 (1989) 2635-2647.

[18] Soo-Jong Rey, "News from Higher Spins: $W_{\infty}$, Black Holes and Entropy", talk given at the workshop "Higher spins and holography", Simons center, March 2011. 